\title{
Romer, Georg
}

Kinder körperlich kranker Eltern: Psychische Belastungen, Wege der Bewältigung und Perspektiven der seelischen Gesundheitsvorsorge Praxis der Kinderpsychologie und Kinderpsychiatrie 56 (2007) 10, S. 870-890

Quellenangabe/ Reference:

Romer, Georg: Kinder körperlich kranker Eltern: Psychische Belastungen, Wege der Bewältigung und Perspektiven der seelischen Gesundheitsvorsorge - In: Praxis der Kinderpsychologie und Kinderpsychiatrie 56 (2007) 10, S. 870-890 - URN: urn:nbn:de:0111-opus-30654 - DOI: 10.25656/01:3065

\section{Vandenhoeck \& Ruprecht}

http://www.v-r.de

\section{Nutzungsbedingungen}

Gewährt wird ein nicht exklusives, nicht übertragbares, persönliches und beschränktes Recht auf Nutzung dieses Dokuments. Dieses Dokument ist ausschließlich für den persönlichen, nicht-kommerziellen Gebrauch bestimmt. Die Nutzung stellt keine Übertragung des Eigentumsrechts an diesem Dokument dar und gilt vorbehaltlich der folgenden Einschränkungen: Auf sämtlichen Kopien dieses Dokuments müssen alle Urheberrechtshinweise und sonstigen Hinweise auf gesetzlichen Schutz beibehalten werden. Sie dürfen dieses Dokument nicht in irgendeiner Weise abändern, noch dürfen Sie dieses Dokument für öffentliche oder kommerzielle Zwecke vervielfältigen, öffentlich ausstellen, aufführen, vertreiben oder anderweitig nutzen.

Mit der Verwendung dieses Dokuments erkennen Sie die Nutzungsbedingungen an.

\section{Terms of use}

We grant a non-exclusive, non-transferable, individual and limited right to using this document.

This document is solely intended for your personal, non-commercial use. Use of this document does not include any transfer of property rights and it is conditional to the following limitations: All of the copies of this documents must retain all copyright information and other information regarding legal protection. You are not allowed to alter this document in any way, to copy it for public or commercial purposes, to exhibit the document in public, to perform, distribute or otherwise use the document in public.

By using this particular document, you accept the above-stated conditions of use.

\section{Kontakt / Contact:}

\section{peDOCS}

DIPF | Leibniz-Institut für Bildungsforschung und Bildungsinformation Informationszentrum (IZ) Bildung

E-Mail:pedocs@dipf.de

Internet: www.pedocs.de

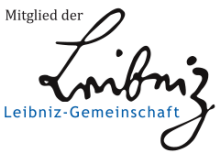




\title{
Kinder körperlich kranker Eltern: Psychische Belastungen, Wege der Bewältigung und Perspektiven der seelischen Gesundheitsvorsorge
}

\author{
Georg Romer
}

\begin{abstract}
Summary
Children of Somatically Ill Parents: Psychological stressors, ways of coping and perspectives of mental health prevention

Irrespective of their well-known increased risk for mental health problems, children of somatically ill parents are a clinically under-served as well as under-researched group. In this article, the author introduces the epidemiologic and clinical relevance of this topic and presents a theoretical framework for understanding the sequelae of a serious parental illness on minor-age children, based on family dynamics and developmental concepts. This is followed by a comprehensive review of current empirical studies in this field, with a special emphasis on results from a transnational European collaborative study (COSIP = Children of Somatically Ill Parents), which was coordinated by the author. The most important recommendations from intervention concepts published to date are summarized. The Hamburg COSIP Concept of Counselling for families with a somatically ill parent is introduced. The author concludes in some outlooks for clinical practice as well as future research.
\end{abstract}

Prax. Kinderpsychol. Kinderpsychiat. 56/2007, 870-890

\section{Keywords}

child of impaired parents - illness and family - psychotraumatology - medical family therapy - psychosocial approaches in family medicine

\section{Zusammenfassung}

Kinder körperlich kranker Eltern gelten als bislang klinisch unterversorgte und zu wenig beforschte Risikogruppe für die Entwicklung von seelischen Gesundheitsproblemen. In diesem Beitrag wird einleitend die epidemiologische und klinische Relevanz der Thematik ausgeführt. Danach gibt der Autor eine umfassende familiendynamische und entwicklungspsychologische Betrachtung der Auswirkungen einer elterlichen körperlichen Erkrankung auf minderjährige Kinder. Daran schließt sich eine Übersicht über aktuelle empirische Studien an, wobei Ergebnisse aus einer vom Autor koordinierten transnationalen europäischen Verbundstudie (COSIP - „Children of Somatically Ill Parents" $)^{1}$ besonders berücksichtigt werden. Die wichtigsten Empfehlungen aus

${ }^{1}$ Dieses Verbundprojekt „Mental Health Prevention in a Target Group at Risk: Children of Somati-

Prax. Kinderpsychol. Kinderpsychiat. 56: 870 - 890 (2007), ISSN 0032-7034

(c) Vandenhoeck \& Ruprecht GmbH \& Co. KG, Göttingen 2007 
bislang publizierten Interventionskonzepten werden zusammengefasst. Das Hamburger COSIPBeratungskonzept für Familien mit einem ernsthaft körperlich erkrankten Elternteil wird vorgestellt. Abschließend werden Ausblicke für Klinik und Forschung diskutiert.

\section{Schlagwörter}

Kinder kranker Eltern - Krankheit und Familie - Psychotraumatologie - Medizinische Familientherapie - psychosoziale Konzepte in der Familienmedizin

\section{$1 \quad$ Einleitung}

Wird in einer Familie mit minderjährigen Kindern ein Elternteil ernsthaft krank, sind hiervon alle Familienmitglieder nachhaltig betroffen. Die Krankheit fällt für den Betroffenen meist in den Lebensabschnitt des mittleren Erwachsenenalters, der in der Regel von maximaler Übernahme von Verantwortung in Familie, Partnerschaft und Beruf geprägt ist. Die Begrenztheit der eigenen körperlichen Existenz wird vom betroffenen Elternteil nicht selten erstmals am eigenen Leib erfahren. Sinnfragen werden neu gewichtet. Bisherige Lebensentwürfe müssen revidiert werden (Romer u. Haagen, 2007). Die Diagnose einer ernsthaften oder gar lebensbedrohlichen Erkrankung, wie beispielsweise Krebs, Multipler Sklerose, AIDS oder dialysepflichtiger Niereninsuffizienz kann bei bislang gesunden Eltern zu einer tiefgreifenden Erschütterung des eigenen Selbstbildes von Unversehrtheit und vitaler Kraft führen (Frick-Bruder, 1998). Mit der Krankheit assoziierte Stressoren wie häufige medizinische Eingriffe, Krankenhausaufenthalte, Veränderungen des körperlichen Erscheinungsbildes und gegebenenfalls die Auseinandersetzung mit einer vitalen Bedrohung werden von allen Familienangehörigen miterlebt (Worsham, Compas, Sydney, 1997; Christ, 2000a; Romer et al., 2002b). Anders als im höheren Alter, in dem Erfahrungen mit Krankheit von vielen geteilt werden, sind Eltern minderjähriger Kindern psychisch meist nicht auf diese Situation vorbereitet. Dies wirkt sich auch auf die Elternfunktion aus. Kranke Eltern fühlen sich häufig in ihrer Elternrolle verunsichert oder zusätzlich belastet durch die Vorstellung, ihre Kinder könnten unter der

cally Ill Parents" wurde von der EU im 5. Rahmenprogramm "Quality of Life" gefördert (Projektnummer QLGT-CT-2001-02378) und vom Autor koordiniert. Die Leiter der anderen beteiligten Forschungsgruppen (principal investigators) waren: Prof. Max Friedrich, Abteilung für Neuropsychiatrie des Kindes- und Jugendalters, Universität Wien (A); Mikael Thastum, Ph.D., Department of Psychology, University of Aarhus (DK); Prof. Jorma Piha, Child Psychiatry Clinic, Turku University Hospital, Turku (FIN); Prof. John Tsiantis, Department of Child Psychiatry, Athens University Medical School, Athen (EL); Prof. Stefan Milea, Clinic of Child and Adolescent Psychiatry, University of Medicine and Pharmacy „CAROL DAVILA“, Bukarest (RO); PD Barbara Steck, Kinder- und Jugendpsychiatrische Universitäts- und Poliklinik Basel (CH); Maggie Watson, Ph.D., Department of Psychological Medicine, Royal Marsden Hospital, Sutton (UK). 
Situation leiden. Kinder und Jugendliche haben je nach Altersstufe unterschiedliche Möglichkeiten, diese familiäre Situation zu bewältigen. Im günstigen Fall reifen sie an der Situation und entwickeln besondere soziale Kompetenzen im Sinne sozialer Verantwortlichkeit oder Fürsorglichkeit (Romer et al., 2006). Viele Kinder reagieren auf die von ihnen erfühlten Belastungen der Eltern, indem sie sich von ihrer stärksten Seite zeigen und ihre eigenen Sorgen von den Eltern fernhalten. Dies trägt zu der bei kranken Eltern beschriebenen Tendenz bei, seelische Belastungen ihrer Kinder zu unterschätzen (Welch, Wadsworth, Compas, 1996). Dies kann aus verhaltensbiologischer Sicht als eine sinnvolle Priorisierung der Gefährdung des eigenen Organismus bzw. seiner Genesung verstanden werden (Romer, Schulte-Markwort, Riedesser, 2002a). Die Legitimation des Bedürfnisses, die eigene Stabilisierung in den Vordergrund zu stellen, ist daher ein wohlbegründetes Element psychosozialer Betreuungskonzepte für körperlich kranke Erwachsene.

\subsection{Epidemiologische Relevanz}

Ausgehend von den Daten des U.S. National Center for Health Statistics lässt sich grob schätzen, dass in westlichen Industrieländern 5-15\% aller Kinder und Jugendlichen im Laufe ihrer Entwicklung von der Situation betroffen sind, dass ein Elternteil schwerwiegend körperlich erkrankt (Worsham, Compas, Sydney, 1997). In einer neueren repräsentativen Befragung von knapp 2000 Familien mit mindestens einem Kind zwischen 4 und 17 Jahren in Deutschland betrug die Punktprävalenz für eine ernsthafte körperliche Erkrankung eines Elternteils 4,1 \%, wobei in einem Drittel der Fälle eine Krebserkrankung angegeben wurde (Barkmann, Romer, Watson, Schulte-Markwort, 2007). Das erhöhte Risiko für Kinder chronisch- und schwerkranker Eltern seelische Gesundheitsprobleme zu entwickeln, ist seit den 60er Jahren belegt (Rutter, 1966). Gleichwohl gibt es im Gesundheitswesen nach wie vor wenig systematische Ansätze zur gezielten Prävention für diese Risikogruppe. Während im umgekehrten Fall, d. h. bei einer ernsthaften Erkrankung eines Kindes, in der Pädiatrie psychosoziale Betreuungskonzepte stets die Eltern und häufig auch die Geschwister einbeziehen, sind in der Erwachsenenmedizin Kinder als Angehörige von körperlich kranken Eltern bislang nicht selbstverständlich im Blickfeld der Behandler. Wenn man sich vergegenwärtigt, dass aufgrund der Abhängigkeit minderjähriger Kinder von ihren Eltern die kindliche seelische Belastung bei einer ernsthaften elterlichen Erkrankung mit existentiellen Verlustängsten einhergeht, wird deutlich, dass es sich bei dieser bislang wenig entwickelten professionellen Aufmerksamkeit für die psychosoziale Situation von Kindern kranker Eltern um einen Artefakt des medizinischen Versorgungssystems handeln muss. Während Eltern kranker Kinder allein schon in ihrer Rolle als Auftraggeber und Bündnispartner der Behandler präsent sind, spielen Kinder kranker Eltern in klassischen Behandlunssettings der Erwachsenenmedizin in der Arzt-Patient-Beziehung keine Rolle. Kranke Eltern machen somit üblicherweise die Erfahrung, dass sie in ihrer Elternrolle und -funktion von ihren Behandlern nicht gezielt wahrge- 
nommen und angesprochen werden. Sie klammern daher ihrerseits diesen Aspekt der Auswirkungen ihrer Krankheit auf ihr Selbsterleben meist aus der Arzt-Patient-Beziehung aus. In bislang entwickelten Konzepten der medizinischen Familienberatung und -therapie (McDaniel, Hepworth, Doherty, 1992; Cierpka, Krebeck, Retzlaff, 2001) wird zwar versucht, eine komplexe Sicht auf das System Familie in bio-psycho-soziale Ansätze der Patientenversorgung (comprehensive care) zu integrieren, jedoch fehlt hierbei meist eine entwicklungspsychologisch differenzierte Berücksichtigung der kindlichen Erlebnisperspektive. Neuere kindzentrierte Interventionsansätze in der medizinischen Familienberatung und -therapie zielen daher u.a. darauf ab, eine möglichst angstfreie Kommunikation zwischen Eltern und Kindern über vorhandene Ängste und Sorgen zu ermöglichen, die elterliche Kompetenz zu stärken, sowie auf Seiten der Kinder eine altersgerechte kognitive Orientierung zur Situation und eine möglichst aktive Bewältigung zu ermöglichen (Hoke, 1997; Lewandowski, 1992; Christ, 2000a; Davis-Kirsch, Brandt, Lewis, 2003; Rotheram-Borus, Murphy, Miller, Draimin, 1997; Romer u. Haagen, 2007; Übersicht bei Diareme et al., 2007).

\section{Familiendynamische Aspekte}

In einer durch die Lebenszyklen der Generationen vorbestimmten Rollenverteilung wird im familiären Kontext heranwachsenden Kindern das kulturelle Wissen um Krankheit und Vergänglichkeit, sowie auch um Sterben und Tod im idealtypischen Fall dadurch vermittelt, dass irgendwann Großeltern oder auch noch lebende Urgroßeltern krank werden und schließlich eines Tages sterben. Die Eltern stehen dann üblicherweise mitten im Leben und sind den heranwachsenden Kindern als „unverwüstliche" Vertreter der Erwachsenenwelt verfügbar (Romer u. Haagen, 2007). Jede ernsthafte oder gar lebensbedrohliche Erkrankung im mittleren Erwachsenenalter erschüttert hingegen diesen biologisch und kulturell vorgezeichneten familiären Lebensplan. Die Vorstellung betroffener Eltern, den eigenen Kindern bis sie erwachsen sind die eigene Lebenstüchtigkeit voll zur Verfügung stellen zu können, erleidet schmerzliche Einschränkungen. In mehrerlei Hinsicht werden Grundfesten der Eltern-Kind-Bindung bedroht. Die Zukunftsperspektive einer Familie und damit das familiäre Kohärenzgefühl sowie die familiäre Identität werden nachhaltig in Frage gestellt (Geigges, 1996). Familien können an dieser Situation wachsen, sie können vorübergehend zusammenbrechen und sich im Laufe der Zeit wieder erholen. Sie können auch auseinanderfallen, wenn sie den Anforderungen an die Umorganisation der Familienstruktur nicht gewachsen sind (Johnson, 1988).

\subsection{Sorgen und Belastungen}

In einer früheren Übersichtsarbeit über empirische Untersuchungen zum Einfluss einer Krebserkrankung auf das Familienleben hat Lewis (1986) die subjektiven Be- 
lastungen zusammengefasst, die von Krebspatienten und ihren Familienangehörigen am häufigsten berichtet wurden. Diese sind weitgehend auch auf andere ernsthafte elterliche Erkrankungen übertragbar. Die zehn vorrangigsten Stressoren waren: 1 . Gefühle von Angst, Hilflosigkeit oder Wut, die dadurch ausgelöst werden, dass man das kranke Familienmitglied leiden sieht; 2. die körperliche Erschöpfung durch die Beanspruchung in der Pflege des Kranken; 3. ein verstärktes Bedürfnis nach körperlicher Nähe bei gleichzeitig vermindertem Wunsch nach sexueller Intimität innerhalb der elterlichen Paarbeziehung; 4. die Unsicherheit über die Prognose der Erkrankung und damit verbundene Angst, das kranke Familienmitglied könne sterben; 5. Veränderungen der familiären Lebensführung und der Rollenverteilung im Haushalt; 6. die Frage, wie man das erkrankte Familienmitglied am besten trösten könne; 7. existenzielle Fragen, mit denen auch jüngere Kinder in entwicklungsakzelerierter Weise beschäftigt sind (z. B. können 8-Jährige intensiv mit Fragen nach dem Sinn des Lebens beschäftigt sein); 8. divergierende Bedürfnisse innerhalb der Familie, wie körperliche Erschöpfung beim Erkrankten, Vielfachbeanspruchung bei dessen Ehepartner und die Beschäftigung mit existenziellen Fragen beim Kind; 9. finanzielle Engpässe; 10. fehlende Unterstützung der Familie.

\subsection{Coping und Resilienz von Familien}

Auf der Basis eigener Untersuchungen an Familien mit einem an Multipler Sklerose erkrankten Elternteil beschrieb Power (1985) als wichtigsten Prädiktor für eine gelungene psychosoziale Bewältigung auf Seiten des Familiensystems dessen Fähigkeit, Teile des Familienlebens aufrechtzuerhalten, die nicht durch die Krankheit und ihre Auswirkungen beeinträchtigt werden. In Familien, in denen die Anpassung an krankheitsbedingte Belastungen gelungen schien, betonten die Familienmitglieder typischerweise, dass sich das Familienleben keinesfalls nur noch vollständig um den erkrankten Elternteil drehen sollte. Die einzelnen Familienmitglieder waren in der Lage, sich auch um ihre eigenen Bedürfnisse hinreichend zu kümmern und waren an außerfamiliären Aktivitäten beteiligt (ebd.). In den Basler Studien zur Krankheitsbewältigung bei Eltern und Kindern in Familien mit einem an Multipler Sklerose erkrankten Elternteil konnten Steck und Mitarbeiter (Steck, Kappos, Bürgin, 1998; Steck, 2002) aufzeigen, dass insbesondere ein bewusst durchlebter Trauerprozess auf Seiten der erkrankten Eltern über den Verlust der unbeeinträchtigten Vitalität eine gelungene Anpassung aller Familienmitglieder begünstigte. In Familien, in denen diese Trauer von Seiten der erkrankten Eltern verleugnend abgewehrt schien und eine bewusst erlebte Trauerarbeit nicht geleistet worden war, konnten bei den Kinder häufiger eine misslingende Bewältigung der Situation festgestellt werden. In einer umfassenden Literaturübersicht bisheriger Studien zu familiären Anpassungsprozessen an eine elterliche Krebserkrankung extrahierte Rost (1992) folgende fünf typische Reaktionsmuster auf der Ebene des Familiensystems, die sich ebenfalls weitgehend auf andere ernsthafte elterliche Erkrankungen übertragen lassen:

(C) Vandenhoeck \& Ruprecht GmbH \& Co. KG, Göttingen 2007 
- Starke Betonung des familiären Zusammenhalts (Kohäsion). Durch die existenzielle Bedrohung des kranken Elternteils wird das Bindungssystem innerhalb der Familie maximal aktiviert. In Gegenwart von Gefahr und entsprechend situationsangemessener Angst fordern Familienmitglieder voneinander Sicherheit, Halt, Trost und Orientierung ein.

- Isolation gegenüber der sozialen Umwelt. Die Anforderungen der Erkrankung beanspruchen den Patienten und seinen Partner oft derart, dass die Pflege sozialer Außenkontakte darunter leidet. Wenn dies dazu führt, dass Freunde und Nachbarn weniger häufig zu Besuch kommen, wird dies von Kindern als unausgesprochene Botschaft wahrgenommen, dass die Realität der elterlichen Krankheit vor der Außenwelt verborgen werden sollte. Als Folge vermeiden Kinder mitunter ebenso, mit ihren Freunden über die elterliche Erkrankung zu sprechen (Romer u. Haagen, 2007).

- Geringe Flexibilität. In der durch die Krankheit hervorgerufenen Krise greift das Familiensystem auf in der Vergangenheit bewährte Strategien zur Problembewältigung zurück. Für eine Experimentierfreudigkeit beim Finden neuer Lösungsstrategien fehlt der innere „Spielraum“ (ebd.).

- Konfliktvermeidung. Tendenziell versuchen Familienmitglieder Spannungen und Streit aus dem Wege zu gehen. Vorrangiges Motiv hierfür ist die soziale Rücksichtnahme auf den erkrankten Elternteil, dem innerfamiliäre Spannungen nicht zugemutet werden sollen.

- Parentifizierung. Sowohl der kranke Elternteil als auch sein gesunder Lebenspartner werden durch die Krankheit stark in Anspruch genommen. Zwangsläufig werden Kinder in alltägliche Aufgaben, die ansonsten von Eltern übernommen wurden, vermehrt eingebunden. Kinder übernehmen damit mehr Verantwortung für die anderen Familienmitglieder und nehmen beispielsweise Aufgaben in der Versorgung jüngerer Geschwister oder des kranken Elternteiles wahr. Wird ein schwer kranker Elternteil zu Hause gepflegt, werden Kinder aller Alterstufen häufig auch in der pflegerischen Versorgung eingespannt, ein Phänomen, das bisher wenig gesellschaftliche Beachtung fand (Aldridge u. Becker, 1993). Hinzu gesellt sich nicht selten eine vermehrte emotionale Bedürftigkeit kranker Eltern, die bei ihren Kindern Nähe und Trost suchen. Insbesondere wenn kranke Eltern alleinerziehend sind oder wenn der Lebenspartner emotional wenig zur Verfügung steht, können Kinder in eine Partnerersatz-Rolle hineingeraten, in der sie sich primär für die emotionalen Bedürfnisse des kranken Elternteils zuständig fühlen. Parentifizierung muss keineswegs per se schädlich sein. Es kann für die Bewältigung von Ohnmachtsgefühlen hilfreich sein, wenn Kinder etwas Konkretes tun können, das dazu beiträgt, dass es dem kranken Elternteil besser geht (Romer u. Haagen, 2007). Voraussetzung hierfür ist, dass diese konkrete Hilfestellung dem Alter des Kindes angemessen ist und ein Kind nach Erledigung einzelner konkreter Aufgaben sich wieder eigenen altersgemäßen Interessen zuwenden kann. 
Jedes dieser genannten familiären Reaktionsmuster kann für sich betrachtet als adaptive Bewältigungsstrategie verstanden werden. Von den Entwicklungsbedürfnissen heranwachsender Kinder her betrachtet, haben jedoch alle fünf Reaktionsmuster gemeinsam, dass sie geeignet sind, Bestrebungen des Kindes nach Autonomie und Individuation gegenüber dem Familiensystem zu hemmen, was wiederum die Identitätsentwicklung behindern kann (Romer et al., 2002a, b; Pott et al., 2005; Romer u. Haagen, 2007).

\subsection{Die Bedeutung der Großelterngeneration}

Wenn ein Elternteil schwer erkrankt, gerät die idealtypische Rollenverteilung im Mehrgenerationen-System aus den Fugen. Im Zuge der oben beschriebenen wirksam werdenden kohäsiven Kräfte im familiären Beziehungsgefüge kommt es häufig zu einer vermehrten Annäherung zwischen Eltern und Großeltern. Oft werden Großeltern zur Unterstützung im Alltag gebraucht, beispielsweise um die Enkelkinder während eines längeren Krankenhausaufenthaltes des kranken Elternteils zu versorgen. Ist hierbei die Beziehung zwischen Eltern und Großeltern tragfähig, was meist eine in der Adoleszenz der Eltern durchlebte gelungene Ablösung voraussetzt, kann diese Wiederannäherung eine Bereicherung für alle drei Generationen sein. Gibt es jedoch ungelöste Ablösungskonflikte zwischen den Eltern und deren Eltern, können diese reaktiviert werden, ohne dass sie ausgetragen werden können. In diesem Fall kann die „Neuauflage“ einer Abhängigkeitsbeziehung für die Familie zur Belastung werden. Großeltern, die in der Krise gebraucht werden, fühlen sich hierdurch in der Regel aufgewertet und stellen ihre Lebenserfahrung gerne zur Verfügung. Gleichzeitig sind sie selbst oft extrem seelisch belastet, insbesondere dann, wenn die elterliche Krankheit lebensbedrohlich ist. Die Vorstellung, das eigene erwachsene Kind möglicherweise zu überleben, bedeutet für Großeltern regelhaft eine schwerwiegende Bedrohung ihres Lebensentwurfes. Zum Schmerz des antizipierten Verlustes des erwachsenen Kindes, dessen unbeeinträchtigte Vitalität dem Menschen im Alter normalerweise hilft, die eigene physische Vergänglichkeit zu akzeptieren, gesellt sich die Angst, selbst möglicherweise nicht gut genug anstelle der jungen Eltern für die heranwachsenden Enkelkinder sorgen zu können. Für minderjährige Kinder sind Großeltern in dieser Situation eine wichtige Verkörperung familiärer Unterstützung, sowie generationenübergreifender Tragfähigkeit familiärer Beziehungen. Wenn Kinder kranker Eltern erleben, dass sich Großeltern mit um die eigenen kranken Eltern kümmern, stellt dies zudem ein Modell dar, bei dem die ältere Generation für die jüngere sorgt. Dies schwächt den potentiellen „Parentifizierungssog“"zur Rollenumkehr zwischen minderjährigen Kindern und ihren Eltern ab.

\section{Entwicklungspsychologische Aspekte}

Abhängig von ihrer kognitiven Reife haben Kinder unterschiedliche Konzepte von Leben und Tod sowie von Krankheit und ihrer Entstehung (Lewandowski, 1992;

(C) Vandenhoeck \& Ruprecht GmbH \& Co. KG, Göttingen 2007 
Christ, 2000a, b). Während jüngere Kinder, insbesondere im Stadium des präoperativen Denkens zwischen zwei und sieben Jahren (Piaget, 1983) Kranksein in erster Linie mit konkret beobachtbaren Merkmalen wie „im Bett liegen“ oder „Medizin nehmen müssen“ verbinden, versuchen Jugendliche, entsprechend dem im zwölften Lebensjahr beginnenden Entwicklungsstadium des formal operativen Denkens (ebd.), Krankheiten nach ihrer Ätiologie und Prognose zu differenzieren. Dieser reflektierende Umgang mit Krankheit führt zwangsläufig dazu, dass Jugendliche mit Fragen nach einer möglichen infektiösen oder genetischen Übertragung der Erkrankung auf sie selbst beschäftigt sind und nach Antworten hierauf suchen (Lewandowski, 1992). Ferner lassen sich für die unterschiedlichen Altersstufen folgende typischen Belastungskonstellationen differenzieren (s. Tab. 1).

Tabelle 1: Altersbezogene seelische Belastungen (aus Romer, Schulte-Markwort u. Riedesser, 2002)

\begin{tabular}{ll}
\hline Typische Belastungen & Zielkonflikt: Leben der Mutter vs. Leben des Kindes \\
\hline Schwangerschaft & Trennung als existentielle Bedrohung \\
Kleinkindalter & Trennung als Bestrafung; Verstümmelungsängste \\
Vorschulalter & Magische Idee, Krankheit verursacht zu haben \\
Schulalter & Körperbezogene Ängste; Angst, die Eltern zu belasten \\
Pubertä t und Jugend & $\begin{array}{l}\text { Angst vor Vererbbarkeit; } \\
\text { Autonomie versus Verantwortung, „Ausbruchsschuld“; } \\
\text { Identitätskonflikte }\end{array}$ \\
\hline
\end{tabular}

- Wird während der Schwangerschaft beispielsweise eine Krebserkrankung diagnostiziert, wird die pränatale Beziehungsaufnahme zwischen Mutter und Kind empfindlich gestört. Die emotionale Besetzung des ungeborenen Kindes kann durch depressive Phasen und Zukunftsängste erschwert werden. Kann eine Chemo- oder Strahlentherapie aus Rücksicht auf den Föten nicht sofort erfolgen, entsteht ein tragischer Zielkonflikt zwischen dem Recht der Mutter auf Überleben und dem Recht des Ungeborenen auf Unversehrtheit. Dies kann verbunden sein mit latenten Vorwürfen von Seiten der Mutter gegenüber ihrem Kind und einer „Überlebensschuld“ des Kindes gegenüber seiner Mutter (Riedesser u. Schulte-Markwort, 1999).

- Ein Säugling (0-12 Monate) erlebt Trennungen von der Mutter, wie sie durch stationäre Behandlungen notwendig werden können, als existenzielle Bedrohung, da er ihre sichere Wiederkehr noch nicht antizipieren kann.

- Das Kleinkind (1-3 Jahre) verarbeitet Trennungen möglicherweise subjektiv als Bestrafung durch Verlassen werden. Werden vertraute Alltagsrituale, die dem 
Kind Sicherheit und Halt vermittelt haben, in der Familienroutine ausgesetzt, kann es zu Entwicklungsrückschritten kommen. Wenn dem Kind keine altersangemessen verstehbare Erklärungen für die im Zuge der elterlichen Krankheit entstehenden konkreten Veränderungen seiner Alltagsrealität oder des körperlichen Erscheinungsbildes gegeben werden, ist es auf seine Phantasien angewiesen (Lewandowski, 1992). Beispielsweise kann der Anblick von Folgeerscheinungen der operativen, chemo- oder strahlentherapeutischen Behandlung eines krebskranken Elternteils wie Amputationen oder Haarausfall, beim Kind, wenn es darauf nicht vorbereitet ist, zu archaischen Verstümmelungsängsten führen. Fantasien, die in diesem Zusammenhang auftreten, sind mitunter bedrohlicher, als es der tatsächlichen medizinischen Realität entspricht.

- Das im magischen Denken behaftete Vorschulkind (3-5 Jahre) kann schuldhafte Fantasien zur Verursachung der elterlichen Erkrankung entwickeln, indem es sich beispielsweise vorstellt, eigene „böse" Gedanken, die Gefühle von Wut und Rivalität gegenüber einem Elternteil begleiten können, hätten diesen krank gemacht. Kinder dieser Altersstufe können ferner den ernsten Gesichtsausdruck bei beiden Eltern als Mitteilung interpretieren, dass Lachen oder Verspieltheit unangemessene oder nicht erwünschte Verhaltensweisen seien (Lewandowski, 1992).

- Schulkinder (6-11 Jahre) denken in sehr konkretem Sinne über mögliche Folgen der elterlichen Erkrankung nach. Dies reicht von Gedanken über krankheitsbedingt nicht stattfindende Urlaubsreisen der Familie bis zur vitalen Bedrohung des erkrankten Elternteils. Die Wahrnehmung, dass der kranke Vater oder die kranke Mutter geschwächt und belastet ist, führt auf der Basis altersentsprechend gereifter sozialer Verantwortlichkeit zu reflektierter Besorgnis (concern) und der Bereitschaft, eigene Bedürfnisse gegenüber den Eltern zurückzustellen. Dies kann dazu führen, dass das Kind verinnerlicht, dass die eigenen Gefühle und Bedürfnisse unwichtig sind. Schulkinder reagieren ferner sehr empfindsam auf wahrgenommene körperliche Veränderungen beim erkrankten Elternteil. Dies kann die Entwicklung des eigenen Körperschemas nachhaltig irritieren (Rost, 1992). Emotionale Belastungen zeigen sich typischerweise in somatischen Beschwerden, wie beispielsweise Bauchschmerzen, Kopfschmerzen oder Einnässen.

- Jugendliche (12-17 Jahre) sind in der Regel sehr bereit, bewusst Verantwortung für den kranken Elternteil, aber auch für die ganze Familie zu übernehmen. Je größer diese Bereitschaft ist, desto stärker können eigene Autonomiewünsche eingeschränkt werden (Lewis, Ellison, Woods, 1985; Riedesser und SchulteMarkwort, 1999). Da altersentsprechend eben diese Autonomie- und Ablösungswünsche erstarken, kann dies wiederum zu ausgeprägten Schuldgefühlen führen („Ausbruchsschuld“ vgl. Seiffge-Krenke, 2001; Geigges, 1996; Lewis et al., 1985; Romer et al., 2002; Riedesser u. Schulte-Markwort, 1999; Hilton u. Elfert, 1996).

(C) Vandenhoeck \& Ruprecht GmbH \& Co. KG, Göttingen 2007 


\section{$4 \quad$ Neuere Studien}

\subsection{Prävalenz psychopathologischer Auffälligkeiten}

In der bereits erwähnten epidemiologischen Untersuchung an knapp 2000 repräsentativ ausgewählten Familien in Deutschland, war die mit der Child Behaviour Checklist (CBCL) gemessene Prävalenz klinisch relevanter psychischer Auffälligkeiten bei Kindern basierend auf einer Falldefinition, die Auffälligkeiten im Grenzbereich einschloss, mit 34 \% doppelt so hoch wie bei den Familien ohne einen körperlich kranken Elternteil, wobei andere elterliche Risikofaktoren wie beispielsweise eine psychische Erkrankung in der Vergleichsgruppe in bevölkerungsrepräsentativem Umfang mit vertreten waren (Barkmann et al., 2007). In einer über gemeindebasierte Krebsregister gewonnenen und damit weitestgehend nicht-selektiven Stichprobe von 66 Kindern mit einem krebskranken Elternteil ergaben sich im Patientenurteil sowie im Selbsturteil der Kinder (ab 11 Jahren) signifikant erhöhte Werte für internalisierende Symptome, nicht jedoch aus der Beurteilerperspektive des gesunden Elternteils. Insgesamt ergaben sich in ca. 50 \% der Fälle Hinweise für psychische Auffälligkeiten (Birenbaum et al., 1999). In einer großen niederländischen Untersuchung an 336 Kindern (Alter: 4-18 Jahre) aus 186 Familien mit einem an Krebs erkrankten Elternteil ergaben sich aus der Beurteilerperspektive des kranken Elternteils sowie im Selbsturteil der über 11-Jährigen gegenüber der zugrunde gelegten Normpopulation bei folgenden zwei nach Altersgruppe und Geschlecht differenzierten Untergruppen auf mehr als das Doppelte erhöhte prozentuale Anteile von mit internalisierenden Symptomen manifest klinisch auffälligen Kindern: a) bei den 4-11-jährigen Söhnen, und b) bei den 12-18-jährigen Töchtern (Visser, Huizinga et al., 2005). In der Hamburger COSIP-Studie an 86 Familien mit einem ernsthaft erkrankten Elternteil (davon 54 mit Krebs und 25 mit MS) wurden Häufigkeit und Art psychischer Symptombildungen bei 4-17-jährigen Kindern untersucht. Es konnte gezeigt werden, dass sowohl im Elternurteil (CBCL) als auch im ab 11 Jahren erhobenen Selbsturteil der Kinder (YSR) die Häufigkeit psychischer Symptombildungen (Gesamtauffälligkeit) mit $31 \%$ (CBCL) bzw. 34 \% (YSR) insgesamt etwa doppelt so hoch war wie in einer Normstichprobe (jeweils $16 \%$ ) mit einer eindeutigen Tendenz zu internalisierender Symptombildung sowie zu vermehrten Auffälligkeiten bei jüngeren Kindern. An spezifischen Symptomen waren somatisierende Beschwerden und ängstlich-depressive Zustände hierbei in allen Altersgruppen am häufigsten (Romer et al., 2007, eingereicht). In einer im Rahmen der COSIP-Verbundstudie rekrutierten transnationalen Untersuchungsgruppe von insgesamt 192 Kindern (4-17 Jahre) aus 144 Familien mit einem an Multipler Sklerose erkrankten Elternteil ${ }^{2}$, lag die Prävalenz klinisch relevanter internalisierender Symptome der Kinder im Urteil des kranken Elternteils mit 23 \% signifikant erhöht im Vergleich zu 16 \% bei einer Normpopulation. Im Selbsturteil der ab 11-jährigen Kinder war sie mit $24 \%$ in vergleichbarem Ausmaß signifikant erhöht (Steck et al., 2007). Erwähnt seien in diesem

(C) Vandenhoeck \& Ruprecht GmbH \& Co. KG, Göttingen 2007 
Zusammenhang zwei Studien mit geringeren Fallzahlen von Familien mit überwiegend krebskranken Müttern ( $\mathrm{n}=22$ bzw. 28), in denen keine signifikant erhöhten internalisierenden Symptomwerte im Vergleich zu jeweils nationalen Normwerten nachgewiesen werden konnten (Hoke, 2001; Heiney et al., 1997). Die Aussagekraft dieser beiden Studien ist jedoch zu relativieren. Bei der erstgenannten Studie (Hoke, 2001) wurden Kinder von Müttern mit Brustkrebs unmittelbar nach bioptischer Diagnosestellung untersucht also zu einem Zeitpunkt, an dem aus Sicht der Kinder sich das Familienleben noch nicht durch die Krankheit wesentlich verändert haben konnte. Bei der zweitgenannten Studie (Heiney et al., 1997) zeigten die Kinder immerhin im Vergleich zur Norm signifikant erhöhte Werte für separat gemessene Angstzustände (state anxiety).

Zusammenfassend ergibt sich vor allem auf der Basis der Studien mit höherer Fallzahl und/oder repräsentativer Aussagekraft bereits bei Querschnittserhebungen eine hinreichende Evidenz für signifikant gehäufte internalisierende Symptombildungen bei Kindern körperlich kranker Eltern, wobei insbesondere jüngere Kinder und adoleszente Mädchen vulnerabel sind. In der Tendenz scheinen bei den über 11-Jährigen, bei denen die Selbstbeurteilerperspektive mit erhoben wurde, diese sensitiver für die Abbildung internalisierender Probleme zu sein. Aufgrund der ausschließlichen Querschnittsdaten kann hingegen keine Aussage darüber gemacht werden, wie hoch der Anteil betroffener Kinder ist, die vielleicht mit einer Latenz von mehreren Jahren nach Diagnosestellung im Laufe ihrer Entwicklung seelische Gesundheitsprobleme bekommen, was einer häufigen Beobachtung in unserer klinischen Praxis entspricht (Romer u. Haagen, 2007).

\subsection{Assoziierte Risiko- und Schutzfaktoren}

In mehreren Studien an Familien mit einem an Krebs erkrankten Elternteil, in denen objektive medizinische Parameter wie Dauer, Staging und Prognose der elterlichen Erkrankung kontrolliert wurden, zeigte sich ein eher geringer bis nicht nachweisbarer Einfluss dieser Faktoren auf die psychische Situation der betroffenen Kinder. Insbesondere die Dauer der Erkrankung schien in keiner der bisherigen Studien eine Rolle zu spielen (Lewis, Hammond, Woods, 1993; Visser et al, 2005; Huizinga et al., 2005b; Howes et al., 1994; Compas et al., 1994; Compas, Worsham, Ey, Howell, 1996; Welch et al, 1996; Watson et al., 2006). Die niederländische Forschergruppe um Visser (Visser et al., 2005; Huizinga et al., 2005) berichtet, dass Kinder und Jugendliche ab 11 Jahren, wenn ein Elternteil an einem Krebsrezidiv erkrankt war, im Selbsturteil höhere psychische Belastungswerte zeigten, als wenn Eltern kein Krebsrezidiv hatten. Insgesamt überwiegen jedoch Studienergebnisse deutlich, in denen medizinische Faktoren keinen nachweisbaren Einfluss auf die Ausprägung psychischer Auffälligkeiten bei Kindern krebskranker Eltern hatten. Auch die bereits erwähnte eigene Hamburger COSIP-Studie konnte keiner-

\footnotetext{
${ }^{2}$ Die Familien dieser Untersuchungsgruppe setzte sich wie folgt aus nationalen Einzelstudien zusammen: Schweiz: $n=66$ (46 \%), Griechenland: $n=53$ (37\%), Deutschland: $n=25$ (17\%).
}

(C) Vandenhoeck \& Ruprecht GmbH \& Co. KG, Göttingen 2007 
lei Einfluss medizinischer Parameter wie Dauer, Schweregrad oder Prognose der elterlichen Erkrankung auf die psychosoziale Situation der Kinder nachweisen mit Ausnahme eines Einflusses der Diagnose selbst. Kinder mit einem an Krebs erkrankten Elternteil zeigten insgesamt, d. h. unabhängig von Schweregrad oder Prognose, in höherem Maße psychopathologische Symptome und eine stärker beeinträchtigte gesundheitsbezogene Lebensqualität als Kinder mit einem an Multipler Sklerose erkrankten Elternteil (Romer et al., eingereicht). Wesentlich deutlichere Effekte auf die psychopathologische Symptombildung von Kindern konnten hingegen für psychosoziale assoziierte Risikofaktoren nachgewiesen werden, insbesondere für elterliche Depression sowie für die Dysfunktionalität familiärer Beziehungsgestaltungen. In Studien zu Kindern krebskranker Eltern zeigte sich, dass erhöhte Depressionswerte bei einem oder beiden Elternteilen deutlich mit erhöhten Symptomwerten bei den Kindern einhergingen (Sigal et al., 2003; Lewis u. Darby, 2003), wobei die erhöhten Symptomwerte für die Kinder meist nur im Elternurteil nachweisbar waren und damit möglicherweise einem durch die Depression bedingten Beurteiler-Bias unterlagen. In der britischen COSIP-Studie konnte in einem multiperspektivischen Design (Eltern, Lehrer und Kinder ab 11 Jahren) an 107 betroffenen Familien mit einem im Frühstadium an Krebs erkrankten Elternteil gezeigt werden, dass psychische Probleme der Kinder mit geringem familiärem Zusammenhalt, mit geringer affektiver Responsivität in den innerfamiliären Beziehungen sowie mit affektiver Verstrickung (affective overinvolvement) zwischen Eltern und Kindern einhergingen. Mütterliche Depression korrelierte mit internalisierenden Symptomen der Kinder vor allem bei den Mädchen (Watson et al., 2006). In der griechischen COSIP-Studie an 56 Familien mit einem an Multipler Sklerose erkrankten Elternteil zeigte sich, dass internalisierende Symptome der Kinder signifikant mit den Depressionswerten der Mütter korrelierten, unabhängig davon, ob diese selbst oder die Väter in den Familien erkrankt waren (Diareme et al., 2006). In der bereits erwähnten multiperspektivisch angelegten transnationalen Untersuchung von 144 Familien aus drei Ländern mit einem an Multipler Sklerose erkrankten Elternteil korrelierte die Depressivität kranker Mütter und noch deutlicher die des gesunden Elternteils mit internalisierenden Auffälligkeiten der Kinder. Waren beide Elternteile depressiv, war der Anteil von mit internalisierenden Symptomen auffälligen Kindern auf das zwei- bis dreifache erhöht im Vergleich zu Familien, in denen kein Elternteil depressiv war (Steck et al., 2007). In Hamburger COSIP-Studie $(n=86)$ korrelierte ebenfalls die Depressivität des gesunden Elternteils mit internalisierenden Symptomen der Kinder. Von allen untersuchten psychosozialen Moderatorvariablen ergab sich jedoch der deutlichste Zusammenhang mit psychopathologischen Symptomen bei den Kindern für die Dysfunktionalität der familiären Beziehungsgestaltung (Romer et al., eingereicht). In einer weiterführenden Untersuchung auf der Basis der Selbsteinschätzungen der 11-17jährigen Kinder $(n=56)$ konnte die Ergebnisse der britischen COSIP-Studie (s. o.) dahingehend bestätigt werden, dass insbesondere zwei Subdimensionen der Familienfunktion zwischen Familien mit symptomauffälligen und asymptomatischen Kindern bzw. Jugendlichen diskriminierten, nämlich: a) die affektive Responsivität und b) die affektive Beziehungsaufnahme. Demnach zeigten Kinder vor- 
wiegend in den Familien internalisierende Symptome, in denen die Familienmitglieder untereinander ihre Gefühle wenig offen ausdrückten und/oder im Sinne affektiver Verstrickung (enmeshment, vgl. Minuchin, Rosman, Baker, 1978) dazu neigten, sich jeweils die Gefühlszustände des anderen zu eigen zu machen oder sich im emotionalen Umgang untereinander einmischend bzw. intrusiv zu verhalten (Romer et al., eingereicht).

Zusammenfassend unterstützt die Studienlage die Annahme, dass objektive medizinische Parameter einer elterlichen Erkrankung wie Dauer oder Schweregrad der physischen Beeinträchtigung eher wenig Einfluss auf die psychischen Auswirkungen der Krankheit auf Kinder haben, wohingegen eine depressive Verarbeitung der Situation insbesondere wenn eine kranke Mütter oder der gesunde Elternteil hiervon betroffen ist sowie eine dysfunktionale affektive Beziehungsgestaltung unter den Familienmitgliedern sich besonders ungünstig auf die psychische Entwicklung der Kinder auswirken. Letztere Erkenntnis ist insofern von klinischer Bedeutung, als die letztgenannten Faktoren durch gezielte psychosoziale Interventionen beeinflusst werden können.

\section{Interventionskonzepte}

Bislang gibt es nur wenige spezielle Interventionsprogramme für Kinder körperlich kranker Eltern. Diese sind in der Regel theoriegeleitet entwickelt worden und meist nicht evaluiert. Bei der Darstellung familientherapeutischer Interventionskonzepte für körperlich Kranke wird in aller Regel nicht besonders Bezug auf die Erlebnisperspektive minderjähriger Kinder genommen. Bei spezifisch kindzentrierten Ansätzen wurden gruppentherapeutische sowie einzeltherapeutische Vorgehensweisen berichtet. Als klinisch-theoretischer Hintergrund wurden u. a. psychodynamische Konzepte (Gunther et al., 1998; Urbach u. Culbert, 1991), kognitiv-verhaltenstherapeutische (Davis-Kirsch et al., 2003; Rotheram-Borus et al., 1997), neuere entwicklungspsychologische oder sozial-kognitive Ansätze (Hoke, 1997; Lewandowski, 1992; Christ, 2000) sowie systemische Konzepte (Dale u. Altschuler, 1999; Rolland, 1999; Sholevar u. Perkel, 1990; Steinglass u. Horan, 1987, 1988) angegeben. Die elterlichen Erkrankungen, zu denen kindzentrierte Interventionsansätze publiziert wurden, beschränken sich vorwiegend auf Krebs, AIDS und Schädel-Hirn-Verletzungen.

\subsection{Wichtige Interventionsziele}

In einer zusammenfassenden Betrachtung bisher publizierter Interventionskonzepte lassen sich die folgenden wichtigsten Ziele kindzentrierter familientherapeutischer Vorgehensweisen nennen (Diareme et al., 2007):

- Ein wichtiges Ziel ist nach Ansicht der meisten Autoren die verbesserte kognitive Orientierung zur Situation der elterlichen Erkrankung. Hierzu werden psychoedukative Vorgehensweisen empfohlen, die darauf ausgerichtet sind, Kindern zu helfen, krankheitsbedingte Veränderungen im Familienleben zu verstehen und 
zu verarbeiten, anstatt sich ihnen ausschließlich passiv oder gar hilflos ausgeliefert zu fühlen.

- $\quad$ Des weiteren gilt als vorrangig, Kindern, die sich vielleicht schon mit ihren Sorgen und Nöten in sich zurückgezogen haben, einen sicheren, Vertrauen stiftenden Rahmen anzubieten, um diese Sorgen auszudrücken.

- Schließlich ist den meisten Empfehlungen gemeinsam, dass Kinder gezielt darin unterstützt werden sollten, etwaige passive Bewältigungsmechanismen zu überwinden (z. B. Vermeidung oder Verleugnung) und aktive Bewältigungsstrategien zu entwickeln, wie beispielsweise die Suche nach sozialer Unterstützung, gezielte Selbstfürsorge, Suche nach zwischenmenschlicher Nähe oder gezieltes Selbstmanagement im Umgang mit negativen Gefühlen.

\subsection{Die Mehrgenerationenperspektive}

In bislang publizierten Interventionsansätzen bleibt die Mehrgenerationenperspektive weitgehend unberücksichtigt, obwohl diese sehr hilfreich sein kann, um transgenerational tradierte innere Repräsentanzen für den Umgang mit krisenhaften Lebensereignissen sowie mit Krankheit, Trauer und Verlust aufzudecken und zu verstehen (Romer u. Haagen, 2007). Diese sind den Eltern oftmals nicht bewusst. In unserer von ökonomischer Sicherheit geprägten spätindustriellen Welt ist es eher die Regel als die Ausnahme, dass Menschen im mittleren Erwachsenenalter sich in ihrem bisherigen Leben kaum oder gar nicht mit einer existenziell bedrohlichen Lebenskrise auseinandersetzen mussten. Insbesondere in der „Nestbau-Phase des familiären Lebenszyklus“ (Frevert, Cierpka u. Joraschky 1996), dem Lebensabschnitt der Familie, in dem Eltern mit jüngeren Kindern zusammen leben, trägt die Anteilnahme an der Entwicklung der Kinder, die meist zusammenfällt mit dem Erreichen von beruflicher Etablierung und ökonomischer Eigenständigkeit, dazu bei, dass zukunftsorientierte Lebensentwürfe im Vordergrund des Selbsterlebens und Weltverständnisses stehen. Die Diagnose einer ernsthaften Krankheit kann diesem „erwachsenen Lebensfrühling“ ein jähes Ende bereiten, für dessen Bewältigung dann keine bewussten inneren Repräsentanzen für den Umgang mit Krise, Gefahr und Verlust zur Verfügung stehen. In diesem Falle werden Bewältigungsmuster vorangegangener Generationen unbewusst übernommen (BoszormenyiNagy u. Sparke, 1981). Dies kann wiederum dazu führen, dass Ehepartner in der Auseinandersetzung mit der neu eingetretenen Situation zu sehr unterschiedlichen individuellen Coping-Strategien greifen und sich dabei gegenseitig nicht mehr verstehen. In der Beratung ist es daher hilfreich, im Elterngespräch eine GenogrammAnamnese zu erheben und gezielt danach zu fragen, welche Schicksalsschläge und Verlusterlebnisse es in der früheren Familienvorgeschichte gegeben hat (z. B. im Zweiten Weltkrieg), und was die heutigen Eltern darüber wissen, wie ihre eigenen Eltern und Großeltern mit diesen Herausforderungen des Schicksals umgegangen sind. Hierdurch können transgenerational tradierte familiäre Coping-Stile aufge- 
deckt und bewusst gemacht werden, was beiden Eltern hilft, ihr eigenes CopingVerhalten sowie das des Partners besser zu verstehen und darüber zu reflektieren (Romer u. Haagen, 2007).

\section{Das Hamburger COSIP-Beratungskonzept}

Das Hamburger COSIP-Beratungskonzept basiert auf den langjährigen Erfahrungen der Beratungsstelle „Kinder körperlich kranker Eltern“ am Universitätsklinikum Hamburg-Eppendorf. Die zugrunde liegenden klinisch-theoretischen Konzepte stammen u. a. aus der Psychotraumatologie (Fischer u. Riedesser, 1999), der Bindungstheorie (Bowlby, 1988) und der psychoanalytischen Familientherapie (Cierpka, 1996; Boszormenyi-Nagy u. Sparke, 1981; Reich, Massing u. Cierpka, 2007) sowie aus den speziell zur kindlichen Verarbeitung einer schweren elterlichen Erkrankung existierenden entwicklungspsychologischen Ansätzen von Lewandowski (1992) und Christ (2000a, b). Um den individuellen, familiären und sozialen Bewältigungsmechanismen und Ressourcen Rechnung zu tragen, setzt das COSIP-Konzept auf unterschiedlichen Ebenen an und verfolgt folgende definierte Ziele (Tab. 2).

Tabelle 2: Die Ziele der Beratung im Hamburger COSIP-Konzept

Familiensystem

- Förderung einer offeneren Kommunikation über die elterliche Erkrankung

- Ermöglichen eines flexibleren Umgangs mit den divergenten Bedürfnissen der einzelner Familienmitglieder

- Reduzierung altersunangemessener Parentifizierung

Elternebene

- Stützung des elterlichen Kompetenzerlebens

- Erhöhung der emotionalen Verfügbarkeit der Eltern

Kindliche Ebene

- Verbesserung des kognitiven Verstehens der elterlichen Erkrankung

- Legitimierung eigener Gefühle und Bedürfnisse

- Unterstützung aktiver Bewältigungsstrategien

- Integration ambivalenter Gefühle

- Unterstützung antizipierender Trauerarbeit

In einem Zeitraum von sechs bis acht Monaten werden in einem den spezifischen Bedürfnissen der Familie angepassten Setting in der Regel drei bis acht Beratungssitzungen angeboten. Diese setzen sich folgendermaßen zusammen (Romer u. Haagen, 2007; Romer et al., 2007).

(C) Vandenhoeck \& Ruprecht GmbH \& Co. KG, Göttingen 2007 


\subsection{Erstgespräch mit den Eltern}

Das Erstgespräch mit den Eltern dient dazu, ein vertrauensvolles Arbeitsbündnis herzustellen. Der Fokus des Gespräches liegt auf den elterlichen Bewältigungsmechanismen, ihrem Erleben von Elternschaft und Elternrolle, der subjektiven Belastung der Eltern und Familie sowie den familiären Ressourcen. Durch gezielte Fragen nach der bisherigen Kommunikation über die Erkrankung im Erwachsenensystem, d. h. innerhalb der elterlichen Paarbeziehung sowie mit engsten Freunden lassen sich die Bedürfnisse der Eltern nach Austausch sowie ihre persönlichen Bewältigungsstile erschließen. Werden diese im Gespräch herausgearbeitet und benannt, gibt dies den Eltern das Gefühl, dass sie in ihrem Krankheitserleben und ihren Coping-Bemühungen vorbehaltlos respektiert und nicht in Frage gestellt werden. Ausgehend von diesem explizit gemachten Modell krankheitsbezogener Kommunikation mit vertrauten Erwachsenen, wird die bisherige „Informationspolitik“ gegenüber den Kindern erfragt, einschließlich aller diesbezüglicher Sorgen der Eltern. Grundsätzlich werden Eltern ermutigt, mit ihren Kindern auf dem gleichen Informationsstand über ihre Krankheit zu kommunizieren wie mit vertrauten Erwachsenen. Die Beratung fokussiert hierbei auf eine altersabhängig kindgerechte Verwörterung krankheitsbezogener Informationen. Ein wichtiges Prinzip ist allerdings, dass alle diesbezüglichen Befürchtungen der Eltern im Vorfeld verstanden und durchgearbeitet werden und dass Eltern darin bestärkt werden, im Zweifelsfalle ihrer intuitiven Einschätzung dessen, was für ihre Kinder angemessen ist, zu folgen.

\subsection{Gespräche mit Kindern und Jugendlichen}

Sind Eltern damit einverstanden, dass ihre Kinder vorgestellt werden, werden diesen ab ca. dem Alter von drei Jahren Einzelsitzungen angeboten. In Familien mit mehreren Kindern finden in der Regel zunächst getrennte Gespräche statt mit der Option, an einem Folgetermin die Geschwister zu einem gemeinsamen Termin einzuladen. Es hat sich bewährt, jedem Geschwisterkind zunächst einen geschützten Raum anzubieten, in dem es über bislang vor den anderen Familienmitgliedern verborgen gehaltene Ängste und Sorgen sprechen kann, ohne befürchten zu müssen, dass diese vor der Familie "geoutet“ werden. Das getrennte Setting ermöglicht zudem, auf die Erlebnisweisen des Kindes altersgerecht einzugehen. Im Vordergrund steht die Exploration der kognitiven Orientierung des Kindes zur elterlichen Erkrankung und ihrer Behandlung sowie die Entwicklung eines altersgerechten kohärenten Narrativs zu den Auswirkungen der elterlichen Krankheit auf das Familienleben. Hierbei wird u. a. das kindliche Ätiologieverständnis der Krankheit erfragt. Dies gibt Aufschluss über kindliche Bedeutungszuschreibungen. Etwaige irrationale Verursachungsmodelle, die mit Schuldgefühlen einhergehen, wie beispielsweise die Vorstellung einer "magischen“ Mitverschuldung der Krankheit durch eigene aggressive Fantasien können erkannt und gegebenenfalls aufgelöst werden. Bislang unbeantwortete oder erstmals gestellte 
Fragen des Kindes können gesammelt und in einem gesondert vorzubereitenden Gespräch mit den Eltern oder dem behandelnden Arzt geklärt werden.

\subsection{Familiengespräche}

Lassen sich in den getrennten Gesprächen mit Eltern und Kindern beidseitige Wünsche nach mehr Austausch innerhalb der Familie herausarbeiten, wird diesen in Familiengesprächen nachgegangen. Ziel dieser Sitzungen ist es, der Familie zu ermöglichen, in einen offeneren Umgang mit der Krankheit und ihren Auswirkungen $\mathrm{zu}$ treten. Individuelle Bewältigungsstrategien der einzelnen Familienmitglieder werden in Anwesenheit aller Familienmitglieder gemeinsam herausgearbeitet und benannt. Dies ermöglicht, Missverständnisse und Kränkungen innerhalb der Familie, wie z. B. Gefühle des Verletztseins durch das Rückzugsverhalten des kranken Elternteils, aufzuarbeiten und wechselseitigen Respekt für die teils divergenten Bedürfnisse der einzelnen Familienmitglieder zu schaffen. Wünsche für den Umgang miteinander können formuliert und die Familie kann in ihren aktiven Bewältigungsstrategien und Ressourcen gestärkt werden.

\subsection{Kriseninterventionen}

Im Falle eines kritischen Krankheitsverlaufes mit möglicherweise tödlichen Komplikationen und Verkettungen schockierender oder ängstigender Erlebnisse kann eine akute Krisenintervention im Rahmen der stationären medizinischen Behandlung indiziert sein. Durch gezielte psychoedukative Unterstützung des medizinischen Personals und des überlebenden Elternteils soll eine altersangemessene Einbindung der minderjährigen Kinder als Angehörige, die Halt und Orientierung sowie angemessene Informationen brauchen, gesichert werden. So können beim Kind Gefühle von Desorientiertheit oder hilflosem Ausgeliefertsein vermieden werden, was wiederum einer traumatischen Verarbeitung der Stresssituation vorbeugen soll.

Die Ergebnisse der bisherigen Evaluation dieses Interventionskonzeptes verweisen auf eine guten Akzeptanz auf Seiten der Familien in Bezug auf Ergebnis- und Prozessqualität (Paschen et al., 2007). Unter den definierten Zielen der Intervention wurden retrospektiv von den befragten Eltern und Kindern die „Stützung des elterliche Kompetenzerlebens“ bzw. die „Unterstützung aktiver Coping-Stile des Kindes“ jeweils erstrangig im Sinne einer positiven Beratungserfahrung genannt (ebd.).

\section{Ausblick}

Kinder als Angehörige ernsthaft erkrankter Patienten sollten gezielter ins Blickfeld von Klinik und Forschung genommen werden. Hierzu sind zwei wesentliche Perspektivenerweiterungen im medizinischen und psychosozialen Helfer-System vonnö-

(c) Vandenhoeck \& Ruprecht GmbH \& Co. KG, Göttingen 2007 
ten: Erstens sollten erwachsene Patienten mit Kindern von Ihren Behandlern gezielt in ihrer Funktion als Eltern wahrgenommen und angesprochen werden. Zweitens ist innerhalb bestehender familienmedizinischer und medizinisch-familientherapeutischer Betreuungskonzepte eine Erweiterung der systemischen Sichtweise um eine entwicklungspsychologisch fundierte kind-zentrierte Perspektive notwendig.

\section{Literatur}

Aldridge, J., Becker, S. (1993). Children as carers. Archives of Disease in Childhood, 69, 459-462.

Barkmann, C., Romer, G., Watson, M., Schulte-Markwort, M. (2007). Parental Physical illness as a risk for psychosocial maladjustment in children and adolescents: epidemiological findings from a national survey in Germany. Psychosomatics (im Druck).

Birenbaum, L., Yancey, D., Phillips, N., Chand, N., Huster, G. (1999). School-age children's and adolescents adjustment when a parent has cancer. Oncology Nursing Forum, 26, 1639-1645.

Boszormenyi-Nagy, I., Sparke, G. (1981). Unsichtbare Bindungen. Die Dynamik familiärer Systeme. Stuttgart: Klett-Cotta.

Bowlby, J. (1988). A Secure Base: Clinical Applications of Attachment Theory London: Tavistock/Routledge.

Christ, G. (2000a). Healing Children's Grief: Surviving a Parent's Death from Cancer. Oxford: Oxford Universities Press.

Christ, G. H. (2000b). Impact of development on children's mourning. Cancer Practice, 8, 72-81.

Cierpka, M. (Hrsg.) (1996). Handbuch der Familiendiagnostik. Berlin: Springer.

Cierpka, M., Krebeck, S., Retzlaff, R. (2001). Arzt, Patient, Familie. Stuttgart: Klett-Cotta.

Compas, B. E., Worsham, N. L., Epping-Jordan, J. E., Grant, K. E., Mireault, G., Howell, D. C., et al. (1994). When mom or dad has cancer: I. Markers of psychological distress in cancer patients, spouses and children. Health Psychology, 13, 507-515.

Compas, B.-E., Worsham, N.-L., Ey, S., Howell, D.-C. (1996). When mom or dad has cancer: II. Coping, cognitive appraisals, and psychological distress in children of cancer patients. Health-Psychology; 15, 167-175.

Dale, B., Altschuler, J. (1999). "In sickness and in health": The development of alternative discourses in work with families with parental illness. . Journal of Family Therapy, 21, 267-283.

Davis-Kirsch, S. E., Brandt, P. A., Lewis, F. M. (2003). Making the most of the moment: when a child's mother has breast cancer. Cancer Nursing, 26, 47-54.

Diareme, S., Tsiantis, J., Kolaitis, G., Ferentinos, S., Tsalamanios, E., Paliokosta, E., et al. (2006). Emotional and behavioural difficulties in children of parents with multiple scelrosis: A controlled study in Greece. European Child and Adolescent Psychiatry, 15, 309-318.

Diareme, S., Tsiantis, J., Romer, G., Tsalamanios, E., Anasontzi, S., Paliokosta, H., et al. (2007). Mental health support for children of somatically ill parents: A review of theory and intervention concepts. Family Systems \& Health. (in Druck)

Fischer, G., Riedesser, P. (1999). Lehrbuch der Psychotraumatologie. München: Ernst Reinhardt Verlag

Frevert, G., Cierpka, M., Joraschky, P. (1996). Familiäre Lebenszyklen. In M. Cierpka (Hrsg.), Handbuch der Familiendiagnostik (S. 163-194). Heidelberg: Springer.

Frick-Bruder, V. (1998). Pschosomatische Gynäkologie und Geburtshilfe. In S. M. Kentenich H (Hrsg.), Beiträge der Jahrestagung 1994/95. Gießen: Psychosozial-Vlg.

(C) Vandenhoeck \& Ruprecht GmbH \& Co. KG, Göttingen 2007 
Geigges, W. (1996). Familienprozesse bei Krebspatienten. In R. H. Adler, J. M. Herrmann, K. Köhle, O. W. Schonecke, T. v. Uexküll, W. Wesiack (Hrsg.), Uexküll - Psychosomatische Medizin (S. 970-978). München: Urban \& Schwarzenberg.

Gunther, M., Crandles, S., Williams, G., Swain, M. (1998). A place called HOPE: Group psychotherapy for adolescents of parents with HIV/AIDS. Child Welfare, 77, 251-271.

Heiney, S. P., Bryant, L. H., Walker, S., Parrish, R. S., Provenzano, F. J., Kelly, K. E. (1997). Impact of parental anxiety on child emotional adjustment when a parent has cancer. Oncol Nurs Forum, 24, 655-661.

Herriger, N. (1993). Die „unverwundbare“ Familie Belastende Lebensumstände und psychosoziale Immunität The invulnerable family. Stressful living conditions and psychosocial immunity. Soziale Arbeit, 42, 146-152.

Hilton, B. A., Elfert, H. (1996). Children's experiences with mothers' early breast cancer. Cancer Practice, 4, 96-104.

Hoke, L. A. (1997). A short-term psychoeducational intervention for families with parental cancer. Harvard Review of Psychiatry, 5, 99-103.

Hoke, L. A. (2001). Psychosocial adjustment in children of mothers with breast cancer. Psycho-Oncology, 10, 361-369.

Howes, M. J., Hoke, L. A., Winterbottom, M., Delafield, D. (1994). Psychosocial effects of breast cancer on the patient's children. Journal of Psychosocial Oncology, 12, 1-12.

Huizinga, G., Visser, A., Graaf, W. v. d., Hoekstra, H., J.Hoekstra-Weebers. (2005). The quality of communication between parents and adolescent children in the case of parental cancer. Annals of Oncology, 16, 1956-1961.

Johnson, C. (1988). Cancer: A family disruption. Recent results. Cancer Research, 108, 306310.

Lewandowski, L. A. (1992). Needs of children during the critical illness of a parent or sibling. Crit Care Nurs Clin North Am., 4, 573-585.

Lewis, F. M. (1986). The impact of cancer on the family: A critical analyses of the research literature. Patient Education and Counseling, 8, 269-289.

Lewis, F. M. (1996). The Impact of Breast Cancer on the Family: Lessons learned from Children and Adolescents. Cancer and the Family, 271-285.

Lewis, F. M., Darby, E. L. (2003). Adolescent adjustment and maternal breast cancer: a test of the 'faucet hypothesis'. Journal of Psychosocial Oncology, 21, 81-104.

Lewis, F. M., Ellison, E. S., Woods, N. F. (1985). The Impact of Breast Cancer on the Familiy. Seminars in Oncology Nursing, 1(3), 206-213.

Lewis, F. M., Hammond, M. A., Woods, N. F. (1993). The family's functioning with newly diagnosed breast cancer in the mother: the development of an explanatory model. J. Behav. Med., 16, 351-370.

McDaniel, S., Hepworth, J., Doherty, W. J. (1992). Medical Family Therapy (Dt. Ausgabe: Familientherapie in der Medizin, Heidelberg: Carl Auer Verlag, 1997 ed.). New York: Basic Books.

Minuchin, S., Rosman, B. L., Baker, L. (1978). Psychosomatic Families: Anorexia Nervosa in Context. Boston, MA: Harvard University Press.

Paschen, P., Saha, R., Baldus, C., Haagen, M., Pott, M., Probst , P., et al. (2007). Evaluation eines präventiven Beratungskonzeptes für Kinder körperlich kranker Eltern. Psychotherapeut (im Druck).

Piaget, J. (1983). Meine Theorie der geistigen Entwicklung. Frankfurt a.M: Fischer. 
Pott, M., Haagen, M., Baldus, C., Saha, R., Romer, G. (2005). Wenn Mütter an Krebs erkranken: seelische Auswirkungen auf Kinder und präventiver Handlungsbedarf Zentralblatt fur Gynakologie, 127, 114-119.

Power, P. W. (1985). Family coping behaviours in chronic illness: a rehabilitation perspective. Rehabilitation Literature, 46, 78-83.

Reich, G., Massing, A., Cierpka, M. (2007). Praxis der psychoanalytischen Familien- und Paartherapie: Kohlhammer.

Riedesser, P., Schulte-Markwort, M. (1999). Kinder körperlich kranker Eltern: Psychische Folgen und Möglichkeiten der Prävention. Deutsches Ärzteblatt, 96, 2353-2357.

Rolland, J. S. (1999). Parental illness and disability: a family system framework. Journal of Family Therapy, 21, 242-266.

Romer, G., Baldus, C., Haagen, M., Pott, M., Saha, R. (eingereicht). Mental health problems in children of somatically ill parents: Predictors of psychological symptoms and quality of life. Clinical Child Psychology \& Psychiatry.

Romer, G., Barkmann, C., Schulte-Markwort, M., Thomalla, G., Riedesser, P. (2002a). Children of somatically III parents: A methodological review. Clinical Child Psychology and Psychiatry, 7, 17-38.

Romer, G., Haagen, M. (2004). Kinder körperlich kranker Eltern: Bedarf für seelische Gesundheitsvorsorge. Frühe Kindheit, 7, 8-15.

Romer, G., Haagen, M. (2007). Kinder körperlich kranker Eltern. Göttingen: Hogrefe.

Romer, G., Haagen, M., Barkmann, C., Thomalla, G., Schulte-Markwort, M., Riedesser, P. (2004). Präventiver Handlungsbedarf bei einer kinder- und jugendpsychiatrischen Risikogruppe: Kinder körperlich kranker Eltern. Hamburger Ärzteblatt, 3/04, 124-127.

Romer, G., Kienbacher, C., Milea, S., Piha, J., Steck, B., Thastum, M., et al. (Eds.) (2005). Children of Somatically Ill Parents: International Perspectives on Family-oriented Mental Health Prevention. Final Consolidated Report of RTD Project QLG4-CT-2001-02378. University of Hamburg: Report to the European Commission.

Romer, G., Möller, B., Haagen, M., Quitman, J., Riedesser, P. (2007). Psychische Belastungen und ihre Bewältigung bei Kindern krebskranker Eltern. Jahrbuch für Medizinische Psychologie (im Druck).

Romer, G., Saha, R., Haagen, M., Pott, M., Baldus, C. (eingereicht). Differential family functioning, family coping and teenage children's psychological adjustment when a parent is physically ill: A multi-perspective study. Family Process.

Romer, G., Schulte-Markwort, M., Riedesser, P. (2002b). Kinder körperlich kranker Eltern am Beispiel Kinder krebskranker Mütter. Geburtshilfe und Frauenheilkunde, 62, 537-542.

Romer, G., Stavenow, K., Brüggemann, A., Baldus, C., Barkmann, C., Riedesser, P. (2006). Kindliches Erleben der chronischen körperlichen Erkrankung eines Elternteils: Eine qualitative Analyse von Interviews mit Kindern dialysepflichtiger Eltern. Praxis der Kinderpsychologie und Kinderpsychiatrie, 55, 53-72.

Rost, R. (1992). Psychosoziale Probleme von Kindern körperlich kranker Eltern- ein Literaturüberblick. Unpublished Medizinische Dissertation, Albert-Ludwigs-Universität Freiburg.

Rotheram-Borus, M. J., Murphy, D. A., Miller, S., Draimin, B. H. (1997). An intervention for adolescents whose parents are living with AIDS. Clinical Child Psychology and Psychiatry, 7, 17-38.

Rutter, M. (1966). Children of sick parents. An environmental and psychiatric study. (Vol. 16). London: Maudsley Monographs. 
Seiffge-Krenke, I. (2001). Diabetic adolescents and their families: Stress, coping, and adaptation. New York: Cambridge University Press.

Sholevar, G. P., Perkel, R. (1990). Family systems intervention and physical illness. GeneralHospital-Psychiatry; 12, 363-372.

Sigal, J. J., Perry, C., Robbins, J. M., Gagne, M., Nassif, E. (2003). Maternal preoccupation and parenting as predictors of emotional and behavioral in children of women with breast cancer. Journal of Clinical Oncology, 21, 1155-1160.

Silverman, P. S. (1999). Never Too Young to Know: Death in Children's lives. Oxford: Oxford Universities Press.

Sperling, E. (1983). Beobachtungen in Familien mit chronischen Leiden. Familiendynamik, $8,32-47$.

Steck, B. (2002). Multiple Sklerose und Familie: Psychosoziale Situation und Krankheitsverarbeitung. Basel: Karger.

Steck, B., Amsler, F., Grether, A., Schwald-Dillier, A., Baldus, C., Haagen, M., et al. (2007). Mental health problems in children of somatically ill parents, e.g. multiple sclerosis. European Child and Adolescent Psychiatry.

Steck, B., Amsler, F., Kappos, L., Burgin, D. (2000). Gender-specific differences coping with chronic somatic disease (e.g. multiple sclerosis). Arch women's ment health. Archives of Women's Mental Health, 3, 15-21.

Steck, B., Kappos, L., Bürgin, D. (1998). Psychosoziale Auswirkungen Multipler Sklerose auf Familie und Kinder. Schweizer Archiv für Neurologie und Psychiatrie, 150, 161-168.

Steinglass, P., Horan, M. (1988). Families and chronic medical illness. In F. Walsh \& C. Anderson (Eds.), Chronic disorders and the family. New York: Harworth Press.

Steinglass, P., Horan, M.-E. (1987). Families and chronic medical illness. Journal of Psychotherapy and the Family, 3, 127-142.

Urbach, J. R., Culbert, J. P. (1991). Head-injured parents and their children: Psychosocial consequences of a traumatic syndrome. Psychosomatics, 32, 24-33.

Visser, A., Huizinga, G., Hoekstra, H., Van der Graaf, W., Klip, E., Pras, E., et al. (2005). Emotional and behavioural functioning of children of a parent with cancer: A cross informant perspective. Psycho-Oncology, 14, 746-758.

Watson, M., St. James-Roberts, I., Ashley, S., Tilney, C., Brougham, B., Edwards, L., et al. (2006). Factors associated with emotional and behavioural problems among school age children of breast cancer patients. British Journal of Cancer, 94, 43-50.

Welch, A. S., Wadsworth, M. E., Compas, B. E. (1996). Adjustment of children and adolescents to parental cancer: Parents' and childrens' perspectives. Cancer, 77, 1409-1418.

Worsham, N. L., Compas, B. E., Sydney, E. Y. (1997). Children's coping with parental illness. In W. Sandler (Ed.), Handbook of children's coping: Linking theory and intervention. New York.

Korrespondenzadresse: PD Dr. med. Georg Romer, Klinik für Kinder- und Jugendpsychiatrie und Psychotherapie, Universitätsklinikum Hamburg-Eppendorf, Martinistr. 52, 20246 Hamburg; E-Mail: romer@uke.uni-hamburg.de 